\title{
MedienPädagogik
}

Zeitschrift für Theorie und Praxis der Medienbildung

Themenheft Nr. 44: Datengetriebene Schule.

Forschungsperspektiven im Anschluss an den 27. Kongress der DGfE

Herausgegeben von Mandy Schiefner-Rohs, Sandra Hofhues und Andreas Breiter

\section{Editorial: Datengetriebene Schule}

\author{
Mandy Schiefner-Rohs ${ }^{1}$ (D), Sandra Hofhues ${ }^{2}$ (D) und Andreas Breiter ${ }^{3}$ (D) \\ ${ }^{1}$ Technische Universität Kaiserslautern \\ ${ }^{2}$ FernUniversität in Hagen \\ ${ }^{3}$ Institut für Informationsmanagement Bremen $\mathrm{GmbH}$, Universität Bremen
}

\section{Einleitung}

Unter medienpädagogischer Perspektive werden mit Daten diverse Forschungsperspektiven aufgeworfen sowie Gestaltungsfragen gestellt, die (nicht nur) den Bildungskontext Schule unmittelbar betreffen. Daten beschreiben nicht nur soziale und demnach auch pädagogische Wirklichkeiten, sie können diese infolge ihrer blossen Verfügbarkeit und Orientierung daran erschaffen, konstruieren und verändern. Zudem sind Software bzw. deren Code sowie Dateninfrastrukturen nicht neutral - es werden soziale Relationen und Ungleichheiten darin fortlaufend technisch eingeschrieben (Dalton und Thatcher 2014; Fuller 2008; Kitchin und Lauriault 2014; Lachney, Babbitt und Eglash 2016, Hartong 2020). Daten präfigurieren damit auch die Bildungsorganisation Schule, so dass beispielsweise nach konkreten Angebotsstrukturen und Massnahmen im Umgang mit Daten und der Reflexion über die Datengesellschaft in der Schule gefragt werden kann. Zudem lässt sich eine zunehmende Verhaltenssteuerung durch Algorithmen beobachten (z. B. Manolev, Sullivan, und Slee 2019), die unter Einbezug ihrer Bezugsdisziplinen medienpädagogisch zu reflektieren ist.

Aktuelle Forschungsarbeiten und nicht zuletzt unsere eigenen Beobachtungen zeigen die Notwendigkeit interdisziplinärer Forschung und Entwicklung im Schulkontext, so dass u. a. im Zusammenspiel von (Medien-)Pädagogik und Informatik vielfältige Forschungsfragen im Feld adressiert werden können (u. a. Breiter und Jarke 2019). Die Bearbeitung des Forschungsfeldes zeigt allerdings einerseits die notwendigen Kooperationen zwischen Wissenschaft, Politik, Verwaltung, Bildungsorganisationen sowie weiteren Akteurinnen und Akteuren von Schule, andererseits sind diese Kooperationen vor dem Hintergrund wissenschaftlichen Wissens über Datenproduktion und -konsum stets kritisch zu analysieren, zu beobachten und/oder zu befragen (vgl. Hartong 2016). (Inter-)national lässt sich hier unmittelbar an die Forschung zur Bedeutung sozialer Vermessungspraktiken, Datafizierung und Algorithmen im Bildungssektor anschliessen (z. B. Boyd und Crawford 2012; Espeland und Stevens 2008; van Dijk 2014; Kitchin 2016; Selwyn 2016; Knox et al. 2019). 
Der hier sehr knapp skizzierte Forschungsstand sowie aktuelle Entwicklungen bringen uns dazu, unserem Themenheft zu «datengetriebener Schule» zwei Prämissen zugrunde zu legen: Erstens gehen wir von einer zunehmenden Relevanz von Daten aus, die unter Rückgriff auf unterschiedliche Tendenzen im Bildungsbereich Auswirkungen auf die Gestaltung von Lern- und Bildungsprozessen in der Schule haben. Zweitens nehmen wir an, dass dieser Prozess subjektivierungstheoretisch zu lesen ist und damit alle Akteurinnen und Akteure in Bildungsorganisationen von datenbezogenen Entwicklungen zwangsläufig betroffen sind.

Datafizierung wird vor diesem Hintergrund einerseits Teil von Organisationskultur und pädagogisch motivierten Schulentwicklungsprozessen, zu denen datenbasierte Kommunikationsstrukturen, Teamentwicklungen und Anforderungen an das Wissensmanagement zählen. Andererseits gilt es, diese Entwicklungen kritisch einzuordnen, insbesondere dann, wenn Daten losgelöst vom Entstehungskontext in permanenter Verbesserungslogik und -rhetorik in der Bildung eingesetzt werden (sollen), wie beispielsweise der Diskurs um Learning Analytics verdeutlicht.

\section{Gegenstandsbereiche des Themenheften und ein Ordnungsversuch}

Bei der Konzeption unseres Themenhefts hatten wir folgende Gegenstandsbereiche besonders im Blick (siehe Call for Papers ${ }^{1}$ ): Neben technologisch-medialen Perspektiven sind für uns - bezogen auf Bildungsprozesse in und mit Datenbezug - auch gesellschaftlich-kulturelle sowie Interaktionsperspektiven relevant, wie dies in Anlehnung an das Frankfurt-Dreieck (2019) auch inter- und transdisziplinäre Sichtweisen von Medienpädagogik, Kommunikations- und Medienwissenschaft und Informatik zum Ausdruck bringen. Wir setzen diese drei Sichtweisen im Folgenden ein, um die dem Themenheft zugrunde liegenden Beiträge aus unser Herausgebenden-Sicht einzuordnen.

Zur technologisch-medialen Perspektivierung gehört, die Materialitäten von Digitalisierung, also beispielsweise Phänomene und Artefakte der durch Digitalisierung geprägten Welt, zu untersuchen und auf diese Weise zugrunde liegende Strukturen und deren Funktionsweise zu reflektieren. Es gilt speziell in unserem Kontext die verheissungsvollen Versprechen individualisierter Lernangebote in den Blick zu nehmen. Automatische Lernanalysen («Learning Analytics») mit dem Versprechen individualisierter bzw. personalisierter Lernangebote finden sich im Bildungskontext Schule ebenso wie in der Lehrpersonenbildung, Hochschule und Weiterbildung. In allen Bereichen sind die dort erzeugten Daten potenziell verknüpfbar mit anderen Datenbeständen und -sätzen - sie erlauben vermeintlich «Vorhersagen» über z. B. Lernentwicklung, Lernerfolg oder auch Lernstrategien. So würde die Verbindung von Daten aus Learning Management Systemen z. B. mit geobasierten Standortdaten der

1 https://www.medienpaed.com/announcement/view/15. 
Lernenden oder Datenspuren in Apps oder Sozialen Medien es zulassen, Vorhersagen über Lern- und/oder Studienerfolg zu treffen (weiterführend Hartong 2019). Medienpädagogisch zu prüfen sind infolgedessen die Grenzen solcher Versprechen und Vorhersagen sowie die Rollen der daran beteiligten (Lehr-)Personen. Wir fragen uns nicht zuletzt: Welche (Vor-)Urteile sind in Daten und Algorithmen eingeschrieben («algorithmic bias»)? Welche Massnahmen zur Reduzierung von Ungleichheiten können bereits technisch, aber auch organisational getroffen werden?

Unter gesellschaftlich-kultureller Perspektivierung werden vor allem Wechselwirkungen zwischen Subjekten, Gesellschaft und technischen Systemen relevant. In den Blick geraten soziale Einschreibungen und Relationalitäten, die vor dem Hintergrund einer tiefgreifend mediatisierten Gesellschaft (Hepp, 2018) und des digitalen Wandels analysiert und reflektiert werden. Medienpädagogisch wird hier vor allem Selbstoptimierung im Lichte von Selftracking fokussiert. Die eigenverantwortliche Sammlung und Auswertung von Daten mittels eigener, zum Teil am Körper getragener Geräte, eröffnet in Lebenswelt und Alltag ein ambivalentes Feld zwischen neuen Bildungserfahrungen auf der einen und Selbsttechnologien auf der anderen Seite (u. a. Damberger 2019). Neben Selftracking erlauben unterschiedliche Devices eine Form von Selbstermächtigung (Gapski 2015; Damberger und Iske 2017; Biermann und Verständig 2017; Dander 2017, 2018; Rode und Stern 2019; von Felden 2020). Darin liegende Ambivalenzen und Grenzen sind speziell für den Kontext Schule zu benennen, sofern diese «Technologien des Selbst» (Foucault 1993) dort zum Einsatz kommen.

Datafizierung von Schule bedeutet aber auch, die Vermessung organisationalen Handelns virulent werden zu lassen, denn: Zur Unterstützung von Schulentwicklung sowie unter dem Paradigma evidenzbasierter Schulentwicklung (Ackeren et al. 2011) werden Daten erzeugt und ausgewertet, um Wissen über die Stärken und Schwächen der Einzelschule und von Schulen im Vergleich zu generieren - und dies gegenüber bildungstheoretischen und/oder -historischen Sichtweisen in evidenzbasierter, zunehmend in softwaregestützter Art und Weise (z. B. Maag Merki und Altrichter 2010; Thiel et al. 2019). Schulinformationssysteme bergen ambivalente Möglichkeiten: Einerseits legen sie Wissen in und über Unterricht und Schulen offen, andererseits kann die Verknüpfung mit anderen Daten vertiefende Einblicke und Bewertungen der Verwaltungs- und Organisationsstrukturen von Einzelschulen und Schularten ermöglichen und Verwaltungsnarrative fördern. Anzunehmen sind auch hier zahlreiche Implikationen, etwa für organisierte Lernprozesse oder kollektive Arrangements des Organisierens und Lernens in der Schule.

Gegenüber der vorgenannten Sichtweise nehmen Interaktionsperspektiven konkrete Praktiken in den Blick, indem hier zentral wird, was Menschen mit Technologie machen und wie sie sich Daten in der für sie spezifischen Weise aneignen. Im Kontext Schule interessieren hier etwa automatisierte Vermessung des Lernstands von 
Schülerinnen und Schülern für Lehrpersonen: Aus Schulverwaltungssoftware, Stundenplanung, elektronischen Klassenbüchern und/oder digitalen Tests etc. lassen sich diese Datensätze leicht generieren oder zusammenführen. Es ergeben sich daraus aber vor allem ethische Herausforderungen, wenn Macht- und Kontrolltechniken infolge datengestützter Schule angewendet werden. Forschungsseitig zeigen sich in Deutschland im Gegensatz zu anderen Ländern (z. B. UK, NL, USA) zudem Lücken, die in Form theoretisch-konzeptioneller Beiträge zu eruieren wären.

Unter medienpädagogischer Perspektivierung rücken darüber hinaus Möglichkeiten von Medienbildung ebenso wie sogenannte Datenkompetenzen («data literacy») in den Fokus des Interesses (Rat für kulturelle Bildung 2019; Jörissen 2019). Während bildungspolitisch und wirtschaftlich gewollte Konzepte von «data literacy» (z. B. digiCompEDU) vor allem informationswissenschaftlich und/oder methodenorientiert oder als Querschnittsaufgabe an die sogenannten MINT-Fächer angehängt sind, gilt es unserer Einschätzung nach gerade die Verbindungen zu tradierten Konzepten von Medienkompetenz und Medienbildung unter emanzipatorischer und kritischer Perspektive herauszuarbeiten (z. B. Niesyto 2018).

Die für das Themenheft eingereichten Beiträge weisen nun genauer aus, wie interdisziplinäre Akteurinnen und Akteure innerhalb von Medienpädagogik das Spektrum datengetriebener Schule selbst (be-)greifen.

\section{Blick in die Beiträge des Themenhefts}

Michael Viertel, Philipp Krieter und Andreas Breiter (Institut für Informationsmanagement Bremen und Universität Bremen) beschäftigen sich in ihrem Artikel «Da habe ich es dann einfach ausgeschaltet»» mit den Möglichkeiten, datengetriebene Analysen von Lernprozessen sowie dem darin liegenden Dilemma zwischen der Verbesserung von Lernprozessen und des Eindringens in Privatsphäre. Am Beispiel von Musikschulkursen zum Thema Songwriting in hybriden Lehr-/Lernarrangements untersuchen sie das Lernverhalten musikbezogener Inhalte in nicht linear organisierten digitalen Lernumgebungen. Der Beitrag nimmt damit vor allem Fragen aus erstgenannter technologisch-medialer Perspektive nach automatischen Vermessungen von Schülerinnen und Schülern auf. Das Ziel bestand darin, mit umfangreichen Analysemethoden von Log-Protokollen sowie Befragungen die digitalen Spuren der individuellen Lernaktivitäten zu verfolgen, um das Lernverhalten musikbezogener Inhalte umfassend zu erforschen. Die Ergebnisse der qualitativen Interviewstudie mit 15 Teilnehmenden geben nicht nur Einblick in die ambivalenten Positionierungen der Befragten hinsichtlich Selbstbestimmung, Entgrenzung, Vermessung und Kontrolle im Kursverlauf, sondern sie machen auch sichtbar, was alles unter der automatisierten Vermessung von Schülerinnen und Schülern adressiert werden kann. 
Der Beitrag von Andreas Weich, Philipp Deny, Marvin Priedigkeit und Jasmin Troeger (Technische Universität Braunschweig) mit dem Titel «Adaptive Lernsysteme zwischen Optimierung und Kritik» enthält «[e]ine Analyse der Medienkonstellationen» der adaptiven Lernplattform bettermarks aus informatischer und medienwissenschaftlicher Sicht. Die Autorin und Autoren zeigen, was u. a. durch Daten in Software wie das hier zugrunde gelegte Beispiel festgeschrieben wird. Sie arbeiten unter Zuhilfenahme des medienwissenschaftlichen Ansatzes der Medienkonstellationen die informatische Modellierung schulischer Praktiken, Subjekte und Inhalte heraus. Hierdurch wird deutlich, welche Logiken in der Software als Technologie eingeschrieben sind und Praktiken präfigurieren. Damit weisen die Autorinnen und Autoren in ihrer Analyse zum einen auf vorherrschende «Logiken des Computational Thinking, des Solutionismus, der Programmierung iterativer bedingter Schleifen, der profilbasierten Repräsentation von Schülerinnen und Schülern, strikte(r) Lernzielorientierung und hierarchische(r) Machtverhältnisse» (Weich et al. 2021, 22) hin. Zum anderen zeigen sie, dass sich das aus der Medienwissenschaft stammende Verfahren der Medienkonstellationen als tragfähiger Analyserahmen erweist, um das Zusammenspiel aus Materialitäten (z. B. Computertechnologie), Wissen/Praktiken (z. B. Gestaltungsprinzipien, informatische Modelle, didaktische Konzepte), Inhalte (z. B. fachliche Inhalte, Oberflächen und Interfaces des Frontends der Software) und Subjektpositionen zu erforschen. Wir verstehen den Artikel daher ebenso als Beitrag zur technisch-medialen Perspektive, wenngleich weniger die automatische Vermessung von Schülerinnen und Schüler im Fokus liegt.

Annina Förschler, Anouschka Kramer, Sigrid Hartong, Claudia Meister-Scheytt und Jaromir Junne (Helmut-Schmidt-Universität Hamburg) widmen sich in ihrem Artikel «Zur (ambivalenten) Wirkmächtigkeit datengetriebener Lernplattformen» der Analyse des «Antolin»-Leseförderungsprogramms, das in schulischen Kontextenverbreitet ist. Die Autorinnen fragen in Anlehnung an Decuypere (2019) danach, wie durch «Antolin» eine designbasierte, datafizierte und datafizierende digitale Bildungswelt erzeugt wird. Durch die Kombination von verschiedenen Datenerhebungsformen zeigen sie die «Wirkmächtigkeit von Plattformen als Denkinfrastrukturen» (Förschler et al. 2021, 57), d. h. dass sich durch die ausgelösten Ambivalenzen neue Logiken in die Schule einschreiben, die sich auch jenseits der grossen, oft in die schulische Diskussion eingebrachten Plattformen zeigen. Wir lesen diesen Beitrag daher primär unter gesellschaftlich-kultureller Perspektive, wenn unter dem Aspekt verheissungsvoller Versprechen individualisierter Lernangebote einerseits Ansprüche an schulische Datenbildung sichtbar werden und diese latenten Wirkmächtigkeiten andererseits zu kritisieren sind. 
Im Zentrum der theoretischen Analyse von Michael Becker, Ulrike Krein und Mandy Schiefner-Rohs (Technische Universität Kaiserslautern) steht der Umgang mit und die Nutzung von Metaphern im Kontext des Datenhandelns von Leitungspersonen in Schulen. Dahinter steht die Erwartung, dass über metaphorische Konzepte latente Denkweisen, Orientierungen und damit auch Praktiken kommunizierbar werden. Unter Rückgriff auf die sozialwissenschaftliche Metaphernanalyse wird hervorgehoben, dass metaphorische Übertragungen über die individuelle Nutzung hinausgehen und auf kulturellen Denkmustern fussen. Ausgehend von Interviews mit Schulleitungen rekonstruieren die Autorinnen und Autoren vier idealtypische Konzepte der Nutzung von Metaphern über das Datenhandeln als Verwalten, Schützen, Sichtbarmachen sowie Waren austauschen. Mit ihren Ergebnissen machen sie deutlich, dass Datenpraktiken als Wechselwirkung zwischen Individuen, Gesellschaft und digitalen Systemen zu verstehen sind und Datafizierung aus Sicht der Leitungspersonen zu einer zunehmenden Vermessung organisationalen Handelns führen kann. Dies kann gleichfalls gesellschaftlich-kulturell betrachtet werden.

An den vorgenannten Beitrag knüpfen die Autorinnen Maike Altenrath, Sandra Hofhues und Jennifer Lange (FernUniversität in Hagen) mit ihrem Artikel insofern an, als dass sie das Verhältnis von Optimierung, Evidenzbasierung und Datafizierung empirisch in den Blick nehmen und nach der grundsätzlichen Ausrichtung des aktuellen Forschungsdiskurses fragen. Mithilfe eines systematischen Reviews bringen die Autorinnen einerseits Perspektiven kritisch verstandener Medienpädagogik und -didaktik ein, andererseits möchten sie sich dem bereits publizierten Diskurs empirisch aufarbeiten. Ihre Analyse stellt dar, wie sich das Verhältnis von Daten und Schulentwicklung im internationalen Diskurs genau gestaltet. So verweist das systematische Review nicht nur auf eine Reihe internationaler und im Kontext des Themenhefts bedeutsamer Schriften, es werden auch fünf relevante Topoi im Anschluss an die Sichtung 33 (inter-)nationaler Veröffentlichungen ausgemacht. So lässt sich festhalten, dass (1) Daten meist planvoll produziert werden, um sie für Rechenschaftslegung und/oder Schulentwicklung zu nutzen. Die Autorinnen führen weiter aus, dass es (2) einen engen Zusammenhang zwischen Dateninfrastrukturen und Lernmanagementsystemen gibt, zur der (3) professionelle Kompetenzen schulischer Akteurinnen und Akteure ein Schlüssel sind. In den Kontext von Schulentwicklungsdiskursen passt den die Topoi, dass (4) das Führungsverhalten die jeweilige Datennutzung prägt. (5) Die Schul- und Kooperationskultur spielt auch hier eine zentrale Rolle. Über exemplarische Ergebnisse hinaus markiert der an gesellschaftlichen Fragen orientierte Beitrag deutlich die Notwendigkeit zur Zusammenschau von Diskursen, sei es in Bezug auf internationale Forschungsbefunde oder innerhalb von Medienpädagogik, Schulpädagogik und Erziehungswissenschaft selbst. 
Britta Galanamatis und Petra Grell (beide Technische Universität Darmstadt) befassen sich in ihrem Artikel «Dann sind die Menschen Spielbälle der Konzerne» mit Privatheitskonzepten von Lehrpersonen. Sie gehen im Beitrag primär der Forschungsfrage nach, welche Perspektiven Lehrpersonen auf die Privatheit ihrer Schülerinnen und Schüler haben, und begeben sich damit auf eine Interaktions- bzw. Handlungsebene. Neben vorangestellten konzeptionellen Überlegungen im Kontext von Privatheit und Medienpädagogik skizzieren sie Ergebnisse einer qualitativempirischen Studie, die auf Basis von Leitfadeninterviews erzeugt wurden. Zu den Ergebnissen ihrer Befragungsstudie gehört das eher «geringe Bewusstsein für den monetären Wert von Daten», aber auch, dass «Entscheidungen, die auf automatisierten Verarbeitungen beruhen, von Lehrpersonen eher abgelehnt werden». Darüber hinaus stellen die Autorinnen unterschiedliche Aushandlungsprozesse (z. B. Privatheitsbedürfnisse) ebenso wie aufscheinende Widersprüche aus medienpädagogischer Sicht heraus (z. B. pädagogisches Ideal und Datenrealität). Ihr Beitrag endet mit Überlegungen zu Interdependenzen von Privatheit. Sie merken hierzu an, dass eine Zielstellung «der weiteren Auseinandersetzung mit der Privatheits- und Datenschutzforschung mit Blick auf Institutionen (der Bildung, aber auch der Familien) [...] aus medienethischer/-pädagogischer Perspektive darin [besteht], Kindern, Jugendlichen und Heranwachsenden kontextuelle Integrität und eine darauf aufbauende Zukunft zu ermöglichen» (Galanamatis und Grell 2021, 135).

Juliane Jarke und Andreas Breiter (Universität Bremen und Institut für Informationsmanagement Bremen) betrachten in ihrem Beitrag «Die Schule als digitale Bewertungsfiguration?», wobei sie einen Schwerpunkt auf die Soziomaterialität von Algorithmen und Daten und ihr inhärenter Bewertungspraktiken legen. Im Sinne einer Vorannahme unterstreichen die Autorin und der Autor - auch angesichts ihrer Verankerung in der Informatik und damit verbundener, möglicher Zuschreibungen im Kontext Medienpädagogik - zunächst, «dass Daten nicht rein technische Artefakte sind, die einen Einfluss auf soziale Praxis haben, sondern dass Daten innerhalb sozialer Praxis erzeugt, interpretiert und verarbeitet werden» (Jarke und Breiter 2021, 143). Mit ihrem Blick «zwischen> Medienpädagogik und Informatik schauen sie im Anschluss an diese konzeptionellen Überlegungen auf drei Beispiele, die ihrerseits Schule im Sinne einer Bewertungsfiguration verändern. Um den Transformationsprozess zu verdeutlichen, gehen sie auf 1 ) Schule als Lern- und Kommunikationsraum, 2) Schulzeit sowie 3) auf die Vermessung von Lehr- oder Lernsubjekten ein. Aus den drei Beispielen ziehen Jarke und Breiter fünf «ambivalente Konsequenzen», die aus der Befassung mit den genannten Transformationsprozessen resultieren. So würden digitale Bewertungspraktiken u. a. neue Formen der Überwachung und Kontrolle zulassen, aber auch grössere Transparenz und Rechenschaftslegung von Akteurinnen und Akteuren einfordern. Auch daher knüpfen sie ihre Ausführungen an 
die wachsende Steuerungs- und Outputorientierung im Bildungsbereich an, welche unter Aspekten von Schule und/oder Governance erst zum Gegenstand medienpädagogischer Forschung werden.

Franco Rau (Universität Vechta) und Anna Geritan (Technische Universität Darmstadt) fokussieren ein Modellschulprojekt zu «digital souverän agierenden Schulen». Der Beitrag ist infolge des forschungsmethodologischen Ansatzes querliegend zu den bis hierhin eingeführten Perspektiven anzusiedeln. Vor dem Hintergrund sich abzeichnender Schulentwicklungsprozesse skizzieren sie in der Denklogik entwicklungsorientierter Bildungsforschung einerseits Wege von Schulen nach, die sich unter Pandemiebedingungen digitalen Medien gewissermassen annähern mussten. Andererseits betrachten sie die Etablierung digitaler Lernplattformen an einer integrierten Gesamtschule. Mit beiden Teilstudien rücken sie sowohl die digitalen Handlungsspielräume der Akteurinnen und Akteure als auch sogenannte souveränitätseinschränkende Bedingungen in den Blick, die sie u. a. am Umgang mit Aspekten von Datenverarbeitung und Datenschutz ausmachen. Darüber hinaus nimmt der Beitrag die Leitbilder der Kultusministerkonferenz (KMK) auf, welche schulische Medienbildung und Schulentwicklung nicht nur im Zusammenhang mit Daten wiederkehrend präfiguriert und die Bedeutung entwicklungsorientierter Forschung innerhalb von Medienpädagogik und -didaktik neuerlich markiert.

In der Zusammenschau der Beiträge ergibt sich weiterer aktueller Forschungsund Reflexionsbedarf, werden doch darin auch blinde Flecken sichtbar: Neben der allgemeinen kritischen Diskussion über die Generierung von Daten und deren Gewinnung im Kontext Schule ist zweifelsohne zu reflektieren, welche Implikationen dies für Bildungsprozesse ebenso wie für Forschung darüber hat. So erfordert Datafizierung geradezu die Weiterentwicklung empirischer Forschungsmethoden (vgl. Mayerl und Zweig 2016, Fromme et al. 2020). Schliesslich gibt es den dringenden Bedarf, sich als Schule zu Prozessen der Datafizierung zu positionieren, der sicherlich (nicht nur) durch die Corona-Pandemie und der damit zusammenhängenden Digitalisierung von Schule weiter an Bedeutung gewinnen wird.

Wir nehmen dieses Themenheft somit als Möglichkeit der Eröffnung eines Dialoges und danken allen Autorinnen und Autoren sowie Reviewerinnen und Reviewer, ohne deren Engagement diese Ausgabe nicht möglich gewesen wäre. Unser abschliessender Dank gilt der Redaktion der Zeitschrift MedienPädagogik, insbesondere Dr. Klaus Rummler, sowie Dr. Dennis Fuchs für die Unterstützung beim Lektorat. 


\section{Literatur}

Ackeren, Isabell van, Olga Zlatkin-Troitschanskaia, Carmen Binnewies, Marten Clausen, Christian Dormann, Peter Preisendoerfer, Christoph Rosenbusch, und Uwe Schmidt. «Evidenzbasierte Schulentwicklung. Ein Forschungsüberblick Aus Interdisziplinärer Perspektive». Die deutsche Schule 1032 (2011): 170-84.

Boyd, Danah, und Kate Crawford. 2012. «Critical Questions for Big Data». Information, Communication \& Society 15(5): 662-79. https://doi.org/10.1080/1369118X.2012.678878.

Biermann, Ralf, und Dan Verständig, Hrsg. 2017. Das umkämpfte Netz: Macht- und medienbildungstheoretische Analysen zum Digitalen. Wiesbaden: Springer Fachmedien Wiesbaden. https://doi.org/10.1007/978-3-658-15011-2.

Breiter, Andreas, und Juliane Jarke. 2019. «Editorial: the datafication of education». Learning, Media and Technology 44(1): 1-6. https://doi.org/10.1080/17439884.2019.1573833.

Dalton, Craig M., und Jim Thatcher. 2014. Inflated Granularity: The Promise of Big Data and the Need for a Critical Data Studies. Presentation at the Annual Meeting of the Association of American Geographers, Tampa, Fl, April 9,2014. https://doi.org/10.1177/2053951715601144.

Damberger, Thomas. 2019. Bildung im digitalen Zeitalter: Zur pädagogisch-anthropologischen, technischen und medienpädagogischen Dimension des Verhältnisses von Bildung und Digitalisierung. Magdeburg: Otto-von-Guericke-Universität Magdeburg. https://doi. org/10.25673/31963.

Damberger, Thomas, und Stefan Iske. 2017. «Quantified Self aus bildungstheoretischer Perspektive». In Das umkämpfte Netz: Macht- und medienbildungstheoretische Analysen zum Digitalen, herausgegeben von Ralf Biermann und Dan Verständig, 17-36. Wiesbaden: Springer Fachmedien Wiesbaden. https://doi.org/10.1007/978-3-658-15011-2_2.

Dander, Valentin. 2017. "Self-Tracking als Gegenstand medienpädagogischer Jugendarbeit?» merz - medien+erziehung 5 (Self-Tracking. Lifelogging. Quantified Self). https://doi. $\operatorname{org} / 10.21240 / \mathrm{mpaed} /$ diss.vd.11.X.

Dander, Valentin. 2018. «Datendandyismus und Datenbildung. Von einer Rekonstruktion der Begriffe zu Perspektiven sinnvoller Nutzung». In Datenflut und Informationskanäle, herausgegeben von Heike Ortner, Daniel Pfurtscheller, Michaela Rizzolli und Andreas Wiesinger, 113-129. https://doi.org/10.21240/mpaed/diss.vd.05.X.

Espeland, Wendy N., und Mitchell L. Stevens. 2008. «A Sociology of Quantification.» European Journal of Sociology 49(3): 401-36. https://doi.org/10.1017/S0003975609000150.

Foucault, Michel. 1993. Technologien des Selbst. Frankfurt a.M.

Fuller, Matthew. 2008. Software Studies / a lexicon. Cambridge, MA: MIT Press.

Gapski, Harald. 2015. Big Data und Medienbildung. Zwischen Kontrollverlust, Selbstverteidigung und Souveränität in der digitalen Welt. Düsseldorf, München: kopaed. https://www. grimme-institut.de/fileadmin/Grimme_Nutzer_Dateien/Institut/Dokumente/SRdg-nrw_ band03_big-data-und-medienbildung.pdf. 
Hartong, Sigrid. 2016. «Between Assessments, Digital Technologies and Big Data: The Growing Influence of 'Hidden' Data Mediators in Education». European Educational Research Journal 15(5) (September 2016): 523-36. https://doi.org/10.1177/1474904116648966.

Hartong, Sigrid. 2019. «Learning Analytics und Big Data in der Bildung. Zur notwendigen Entwicklung eines datenpolitischen Alternativprogramms». Dokumentation des Vortrags im Rahmen der GEW Arbeitsgruppe ‘Bildung in der digitalen Welt), Fulda, 20.09.2019. GEW Broschüre Bildung in der digitalen Welt. https://bildung-wissen.eu/wp-content/uploads/2019/12/hartong_learning_analytics_2019.pdf.

Hartong, Sigrid. 2020. «The power of relation-making: insights into the production and operation of digital school performance platforms in the US». Critical Studies in Education 1-16. https://doi.org/10.1080/17508487.2020.1749861.

Hepp, Andreas. 2018. «Von Der Mediatisierung Zur Tiefgreifenden Mediatisierung». In Kommunikation - Medien - Konstruktion: Braucht Die Mediatisierungsforschung Den Kommunikativen Konstruktivismus?, herausgegeben von Jo Reichertz and Richard Bettmann, 27-45. Wiesbaden: Springer Fachmedien Wiesbaden. https://doi.org/10.1007/978-3-658-212049_2.

Kitchin Rob, und Tracey P. Lauriault. 2014. «Towards Critical Data Studies: Charting and Unpacking Data Assemblages and Their Work» (July 30, 2014). The Programmable City Working Paper 2; preprint version of chapter to be published in Geoweb and Big Data, herausgegeben von Josef Eckert, Andrew Shears, and Jim Thatcher. University of Nebraska Press. Forthcoming. https://ssrn.com/abstract=2474112.

Lachney, Michael, William Babbitt, und Ron Eglash. 2016. "Software Design in the «Construction Genres of Learning Technology: Content Aware versus Content Agnostic». Computational Culture. http://computationalculture.net/2016/01/11/software-design-in-the-construction-genre-of-learning-technology-content-aware-versus-content-agnostic/.

Kitchin, Rob. 2016. «Thinking critically about and researching algorithms. Information». Communication \& Society 20(1): 1-16. https://doi.org/10.1080/1369118X.2016.1154087.

Knox, Jeremy, Ben Williamson, und Sian Bayne. 2019. «Machine behaviourism: future visions of 'learnification' and 'datafication' across humans and digital technologies». Learning, Media and Technology: 1-15. https://doi.org/10.1080/17439884.2019.1623251.

Maag Merki, Katharina, und Herbert Altrichter. 2016. «Empirische Erforschung schulischer Governance. Eine Zwischenbilanz und offene Forschungsfragen». In Handbuch Neue Steuerung im Schulsystem, herausgegeben von Herbert Altrichter und Katharina Maag Merki, 479-86. Educational Governance. Wiesbaden: Springer Fachmedien. https://doi. org/10.1007/978-3-531-18942-0_18.

Manolev, Jamie, Anna Sullivan, und Roger Slee. 2019. «The datafication of discipline: ClassDojo, surveillance and a performative classroom culture». Learning, Media and Technology 44(1): 36-51. https://doi.org/10.1080/17439884.2018.1558237. 
Mayerl, Jochen, und Katharina A. Zweig. 2016. «Digitale Gesellschaft und Big Data: Thesen zur Zukunft der Soziologie». Berliner Debatte Initial 27(4): 77-83. https://shop.welttrends. de/e-journals/e-paper/berliner-debatte-initial/2016-big-data-als-theorieersatz/digitalegesellschaft.

Niesyto, Horst. 2018. «Medienkritik - Entwicklungslinien und aktuelle Herausforderungen». In Medienkritik im digitalen Zeitalter, herausgegeben von Horst Niesyto und Heinz Moser, 5975. München: kopaed. https://doi.org/10.21240/mpaed/37/2020.07.02.X.

Rode, Daniel, und Martin Stern. 2019. Self-Tracking, Selfies, Tinder und Co. Konstellationen von Körper, Medien und Selbst in der Gegenwart. Bielefeld: transcript. https://doi. org/10.14361/9783839439081.

Selwyn, Neil. 2016. "There's so much data': Exploring the realities of data-based school governance.» European Educational Research Journal 15(1): 54-68. https://doi. org/10.1177/1474904115602909.

Thiel, Felicitas, Jasmin Tarkian, Eva-Maria Lankes, Norbert Maritzen, Thomas Riecke-Baulecke, und Anna Kroupa. 2019. Datenbasierte Qualitätssicherung und -entwicklung in Schulen. Eine Bestandsaufnahme in den Ländern der Bundesrepublik Deutschland. Wiesbaden: Springer VS. https://doi.org/10.1007/978-3-658-23240-5.

van Dijck, José. 2014. «Datafication, dataism and dataveillance: Big Data between scientific paradigm and secular belief». Surveillance \& Society 12(2): 197-208. https://doi.org/10.24908/ ss.v12i2.4776.

Von Felden, Heide. 2020. Selbstoptimierung und Ambivalenz. Gesellschaftliche Appelle und ambivalente Rezeptionen. Wiesbaden: Springer VS. https://doi.org/10.1007/978-3-658-281076.

\section{Beiträge}

Altenrath, Maike, Sandra Hofhues, und Jennifer Lange. 2021. «Optimierung, Evidenzbasierung, Datafizierung: Systematisches Review zum Verhältnis von Daten und Schulentwicklung im internationalen Diskurs». Herausgegeben von Mandy Schiefner-Rohs, Sandra Hofhues, und Andreas Breiter. MedienPädagogik: Zeitschrift für Theorie und Praxis der Medienbildung 44 (Datengetriebene Schule): 92-116. https://doi.org/10.21240/mpaed/44/2021.10.30.X.

Becker, Michael, Ulrike Krein, und Mandy Schiefner-Rohs. 2021. « ... dass man denen auch mal «nen Spiegel vorhalten kann»: Metaphern im Diskurs um Daten (in) der Schule». Herausgegeben von Mandy Schiefner-Rohs, Sandra Hofhues, und Andreas Breiter. MedienPädagogik: Zeitschrift für Theorie und Praxis der Medienbildung 44 (Datengetriebene Schule): 73-91. https://doi.org/10.21240/mpaed/44/2021.10.29.X.

Förschler, Annina, Sigrid Hartong, Anouschka Kramer, Claudia Meister-Scheytt, und Jaromir Junne. 2021. «Zur (ambivalenten) Wirkmächtigkeit datengetriebener Lernplattformen: Eine Analyse des ‘Antolin`-Leseförderungsprogramms». Herausgegeben von Mandy SchiefnerRohs, Sandra Hofhues, und Andreas Breiter. MedienPädagogik: Zeitschrift für Theorie und Praxis der Medienbildung 44 (Datengetriebene Schule): 52-72. https://doi.org/10.21240/ mpaed/44/2021.10.28.X. 
Galanamatis, Britta, und Petra Grell. 2021. «Dann sind die Menschen Spielbälle der Konzerne»: Privatheitskonzepte von Lehrpersonen vor dem Hintergrund der Implementierung digitaler Technologien im Unterricht». Herausgegeben von Mandy Schiefner-Rohs, Sandra Hofhues, und Andreas Breiter. MedienPädagogik: Zeitschrift für Theorie und Praxis der Medienbildung 44 (Datengetriebene Schule): 117-39. https://doi.org/10.21240/mpaed/44/2021.10.31.X.

Jarke, Juliane, und Andreas Breiter. 2021. «Die Schule als digitale Bewertungsfiguration? Zur Soziomaterialität von Algorithmen und Daten». Herausgegeben von Mandy SchiefnerRohs, Sandra Hofhues, und Andreas Breiter. MedienPädagogik: Zeitschrift für Theorie und Praxis der Medienbildung 44 (Datengetriebene Schule): 140-59. https://doi.org/10.21240/ mpaed/44/2021.11.01.X.

Rau, Franco, und Anna Geritan. 2021. «Auf dem Weg zu digital souverän agierenden Schulen: Erste Erfahrungen und Erkenntnisse eines entwicklungsorientierten Modellschulprojektes». Herausgegeben von Mandy Schiefner-Rohs, Sandra Hofhues, und Andreas Breiter. MedienPädagogik: Zeitschrift für Theorie und Praxis der Medienbildung 44 (Datengetriebene Schule): 160-84. https://doi.org/10.21240/mpaed/44/2021.11.02.X.

Viertel, Michael, Philipp Krieter, und Andreas Breiter. 2021. «Da habe ich es dann einfach ausgeschaltet): Perspektiven von Lernenden auf Datensammlung mittels Langzeit-Bildschirmaufzeichnungen in non-formalen Bildungskontexten». Herausgegeben von Mandy Schiefner-Rohs, Sandra Hofhues, und Andreas Breiter. MedienPädagogik: Zeitschrift für Theorie und Praxis der Medienbildung 44 (Datengetriebene Schule): 1-21. https://doi.org/10.21240/ mpaed/44/2021.10.26.X.

Weich, Andreas, Philipp Deny, Marvin Priedigkeit, und Jasmin Troeger. 2021. «Adaptive Lernsysteme zwischen Optimierung und Kritik: Eine Analyse der Medienkonstellationen bettermarks aus informatischer und medienwissenschaftlicher Perspektive». Herausgegeben von Mandy Schiefner-Rohs, Sandra Hofhues, und Andreas Breiter. MedienPädagogik: Zeitschrift für Theorie und Praxis der Medienbildung 44 (Datengetriebene Schule): 22-51. https://doi.org/10.21240/mpaed/44/2021.10.27.X. 